\title{
Possible Worlds for Modal Primitivists
}

\author{
Louis deRosset
}

July 12, 2012

\begin{abstract}
Among the most remarkable developments in metaphysics since the 1950's is the explosion of philosophical interest in possible worlds. This paper proposes an explanation of what possible worlds are, and argues that this proposal, the interpreted models conception, should be attractive to anyone who thinks that modal facts are primitive, and so not to be explained in terms of some non-modal notion of "possible world." I articulate three constraints on any acceptable primitivist explanation of the nature of possible worlds, and show that the interpreted models conception meets the three constraints.
\end{abstract}

\section{Possible Worlds and Modal Primitivism}

Among the most remarkable developments in metaphysics since the 1950's is the explosion of philosophical interest in possible worlds. But what are those possible worlds? Perhaps a clue is provided by the fact that every instance of the schema

(SC) it is possible that $\phi$ iff there is a $w$ such that $w$ is a possible world and ' $\phi$ ' is true at $w$.

is widely assumed to be true. (SC) reveals a systematic correspondence between certain modal facts and facts regarding the existence of possible worlds. To some philosophers, (SC) and associated claims held out the hope of providing an explanation in non-modal terms of those modal facts which can be expressed by idioms representable in quantified modal logic. Some even proposed that all modal facts could be explained in terms of facts about which possible worlds 
there were and what they were like. ${ }^{1}$ Providing a non-modal explanation of the notion of being a possible world has occupied center stage in the thought of these philosophers. It will not, however, occupy center stage here. We are not going to require here that the notion of being a possible world be explained in thoroughly non-modal terms. Suppose, then, that modal primitivism is true: some modal facts cannot be reduced to, or even explained in terms of, any congeries of exclusively non-modal facts.

Even granting that (SC) does not provide fodder for an explanation of modal facts in non-modal terms, there remain a number of alternative views of (SC)'s significance. One could suggest, for instance, that instances of (SC) are simply meaningless, since they all crucially employ the notion of being a possible world, and that notion has not been explained. Alternatively, one could simply deny that there are any possible worlds. Then there will be instances of (SC) - those involving true possibility claims - that are false.

If we take either of these skeptical lines, then much of contemporary modal metaphysics will turn out to be either meaningless or a pack of falsehoods. And other disciplines that make liberal use of possible worlds, e.g. some strands of linguistics, ethics, epistemology, and decision theory, will suffer from the same defect. ${ }^{2}$ This need not imply that talk of possible worlds in all of these disparate fields of inquiry is useless or unenlightening. A theory incorporating (SC) might be a useful or edifying fiction, for instance. ${ }^{3}$ Alternatively, (SC) might be false, even though some close surrogate is true. (SC) on this view would inherit its usefulness from the usefulness of its true surrogate. Still, we might wonder whether a more charitable course is available.

I will argue that there is a more charitable course available. Modal primitivists can provide an explicitly modal explanation of the notion of a possible world that, I will argue, renders instances of (SC) both meaningful and true. Other writers have also advanced proposals for explaining the notion of a pos-

\footnotetext{
${ }^{1}$ (Lewis, 1986)

${ }^{2}$ Examples of theorizing in these disparate fields that relies on possible worlds are abundant; a small sample of pieces includes (Arntzenius, 2003), (Blackburn, 1985), (Lewis, 1979), (Meacham, 2008, 2010), and (Stalnaker, 1978). Perhaps a case can be made that, though modal metaphysics and other disciplines appear to share an ontology of possible worlds, the entities used in the other disciplines are radically different from those mentioned in (SC). I will not be assessing the prospects for this line here. Instead, I will be assuming that appearances are not deceiving in this respect.

3 (Rosen, 1990)
} 
sible world in explicitly modal terms. ${ }^{4}$ Each such explanation, in conjunction with ancillary claims like (SC), generates a theory of possible worlds. Thus, many primitivist theories of possible worlds are available. The purpose of this paper is to propose one such theory. Before I outline the proposed theory, however, I will articulate three constraints on theories of possible worlds.

\section{Constraints on an Acceptable Explanation}

There are three constraints which a theory of possible worlds should meet.

\section{$2.1 \quad$ Existence}

First, the possible worlds should exist according to the theory. ${ }^{5}$ This is required if we are to take all instances of (SC) to be straightforwardly true, since some instances of its left-hand side are true. Hence, this constraint is motivated by the very description of the project whose prospects we are exploring: providing an explicitly modal explanation of the notion of a possible world that does not require that we take a skeptical line on (SC). Call this the constraint of existence.

\subsection{Ontological Modesty}

The second constraint is that our theory should avoid, so far as possible, ontological extravagance. That is, our theory should not, so far as possible, commit us to the view that there are things of a certain sort when, before we came to the theory of possible worlds, we would have thought that there are no such things.

The constraint against ontological extravagance is motivated by the fact that those antecedent opinions may form the basis of objections to our theory of possible worlds. If, for instance, our theory commits us to there being such

\footnotetext{
${ }^{4}$ See, for instance,(Kripke, 1980) (Plantinga, 1973), (Salmon, 1989), (Sider, 2002) and (Stalnaker, 1976).

${ }^{5}$ For the purposes of this paper, I am using 'exist' as a shorter equivalent for 'is among the things that there are.' Hence, ' $x$ exists' in the sense of this paper is equivalent to 'there is a $y$ such that $y=x$.' Further, I am abstracting away from complications introduced by the fact that, for many things, existence is temporary. Thus, in the parlance I am adopting here, "The Colossus of Rhodes exists" remains true, even though the Colossus of Rhodes is no longer among us. I believe that this usage tracks at least one use of "there is/are" in English, which might sometimes get used, e.g. in encyclopedia entries, as in "there are three great ancient Greek philosophers: Socrates, Plato, and Aristotle."
} 
things as talking donkeys, then the claim that there are no such things presents a prima facie objection to the theory. Perhaps every theory of possible worlds will have to indulge in extravagance at one point or another. If so, then this constraint cannot be perfectly satisfied. Even so, those theories that are less extravagant are less susceptible to such objections. Call this second constraint the constraint of ontological modesty.

\subsection{Modal Modesty}

We can call the third constraint on an acceptable theory of possible worlds the constraint of modal modesty: our theory of possible worlds should, as much as possible, commit us to no post hoc conclusions about the non-logical modal facts. Here, for instance, is an evident fact regarding George W. Bush: he might have lost the 2000 electoral vote. We shouldn't endorse a theory of possible worlds that commits us to the claim that he could not have lost. Of course, we might be wrong about what the modal facts are, so we should be open to an argument that, in this or that case, our common-sense description of modal reality is wrong. But, in the absence of a compelling argument, e.g. that Bush could not have lost, the claim that he might have lost provides the basis for an objection to any theory which contradicts that claim.

But the constraint of modal modesty applies even in cases in which the modal facts are under serious dispute. The constraint is motivated in these cases, not by the need for a materially adequate theory which avoids counter-example, but also by methodological considerations. Our theory of possible worlds should provide a neutral framework within which to carry on non-logical disputes about what's possible. These issues, then, should not be decided by the metaphysics and logic of the framework. As with the case of ontological modesty, it may be impossible for our theory of possible worlds to be completely neutral on the non-logical modal facts. But we can at least aspire to as much neutrality as may be had.

\subsection{The Significance of the Three Constraints}

Our assumption of modal primitivism implies that possible worlds cannot be used to provide an analysis or reduction of modal claims or facts to non-modal terms. But, as I've noted, the utility of possible worlds is not limited to this 
reductive project in the metaphysics of modality. Possible worlds are useful in a wide variety of theoretical endeavors far removed from the metaphysics of modality. Call the theorists who rely on (SC) and related claims in theorizing in areas other than the metaphysics of modality, the semantics of modal discourse, or the analysis of modal concepts end-users for theories of possible worlds.

Meeting the three constraints underwrites the usefulness of possible worlds for these end-users' theoretical endeavors. Conversely, violating the three constraints undermines the usefulness of possible worlds for end-users. If we endorse a theory of possible worlds that violates the existence constraint, for instance, then we cannot interpret theorizing that relies on (SC) as literally true on the most straighforward reading. If we endorse a theory of possible worlds that violates ontological modesty by endorsing implausible ontological claims, then we export those implausibilities to end-users' theories. Likewise, violating modal modesty holds their theorizing hostage to abstruse disputes in modal metaphysics. Each of these features makes possible worlds less serviceable for theoretical projects outside the metaphysics of modality. It would be better to avoid them if we can.

\subsection{The Three Constraints in Action}

The three constraints combine to make the problem of explaining the notion of being a possible world rather more difficult than one might have hoped. Suppose we explain "possible world" on the model of such ordinary, and straightforwardly modal, notions as "possible Republican nominee for president in 2012." It is plausible to hold that someone is a possible Republican nominee for president in 2012 just in case it is possible that she be a Republican nominee for president in 2012. Sarah Palin, Mitt Romney, and Tim Pawlenty are all possible Republican nominees for president in 2012. The gas station around the corner is not a possible Republican nominee for president in 2012.

Suppose we explain the notion of being a possible world along similar lines: $x$ is a possible world iff it is possible that $x$ be a world. ${ }^{6}$ Such a view will have difficulty simultaneously meeting all three constraints.

First, apply the modal modesty constraint. We take "it is possible that Bush lost the 2000 electoral vote" to be true and so part of our common-sense description of the modal facts. (SC) requires that there be a possible world such

${ }^{6}$ See (Fine, 1985, 2003b) for a defense of a view along these lines. 
that "Bush lost the 2000 electoral vote" is true at that world. The world in which we live is a possible world, according to the conception we are considering, but "Bush lost the 2000 electoral vote" is not true at that world. So modal modesty seems to require that some $w$ is a possible world distinct from the actual world.

Now apply the existence constraint. Perhaps it is plausible to hold that there might have been a world distinct from our world. But the analysis cannot appeal to the truth of "Bush lost the 2000 electoral vote" in $w$ if $w$ is something that doesn't exist, but might have. So the constraint requires that there be a $w$ among the things that exist that is a possible world, and is distinct from the actual world.

Finally, apply the ontological modesty constraint. We are antecedently inclined to think that there are no worlds other than the actual world. This constraint, then, requires that our $w$ be a possible world, but not a world. At first, this might seem to be perfectly fine. Someone can be a possible Republican nominee for president in 2012 without being a Republican nominee. Why, then, can't something be a possible world without being a world? The problem is that we are antecedently inclined to deny that there is an individual that might have been a world, but isn't. The view we are considering faces a prima facie problem, then, satisfying all three constraints.

As the example illustrates, violations of these constraints can often be traded off for one another. Modal modesty can sometimes be secured with ontological extravagance. We could secure the truth of "Bush might have lost the 2000 electoral vote," for instance, by admitting entities that might have been worlds, but are not. Ontological modesty can sometimes be bought by allowing ourselves reference to non-existent worlds. ${ }^{7}$ And we could embrace the theory of possible worlds we have been discussing without ontological extravagance, if we could swallow the extreme modal extravagance of Spinozistic necessitarianism. Then there would be only one possible world, the world we all inhabit. ${ }^{8}$

\footnotetext{
${ }^{7}$ This is the response favored by (Fine, 1985, 2003b).

${ }^{8}$ The difficulty of meeting all three constraints is underscored by the fact that, arguably, extant primitivist theories do not. For instance, some theorists propose to violate the constraint of existence by denying some instances of (SC), interpreted literally and at face value. See, for instance, (Fine, 1985, 2003b), (Salmon, 1989, pp. 17-8n.). Other primitivst theories have been accused of accepting ontological extravagance. Bennett (2005) and Fine (1985) argue that this is the case for Plantinga's view; see esp. (Plantinga, 2003, pp. 116-7). Bennett's (2005, p. 317) discussion of this problem suggests that she proposes instead to pin a substantial modal commitment on end-users, violating modal modesty.
} 
Of course, failure to meet one of the constraints does not constitute a decisive objection against a theory. But this concession should not obscure the fact that a proposal that meets one of the constraints is to that extent preferable to one that does not. And we can always hope for perfection: an ideal proposal that meets all of these constraints.

\section{$3 \quad$ Interpreted Models and Modal Facts}

We have seen that a common sense explanation of the notion of being a possible world has difficulty meeting our three constraints. If we want a theory of possible worlds that does a better job at meeting the three constraints, we should stop treating "possible world" as a bit of ordinary English on the model of "possible Republican nominee." We should, instead, treat it as a technical theoretical notion.

What interpretation, then, of the technical vocabulary "possible world" should we give? Well, undoubtedly the enthusiasm for (SC) and other applications of possible worlds theories is explained in part by the success of Kripkean model theories for modal logics. This is where I propose to start looking. I will assume that the sort of model which we will be dealing with is a Kripke-model. ${ }^{9}$ For a given language, each such model has a model structure $\langle w, W, R\rangle$, with $W$ a non-empty set (thought of as a set of "possible worlds"), $w$ a member of that set (thought of as "the actual world"), and $R$ an accessibility relation on the set. I will say that $x$ is an element of a model $M$ iff $M$ is a model with model structure $\langle w, W, R\rangle$, and $x$ is a member of $W$. The model assigns a domain of objects to every one of its elements, extensions to every predicate-element pair, and some object from some domain of some element to every individual constant. The domains of different elements of $W$ may overlap, and truth in the model is defined in terms of satisfaction in some standard way. The view I propose is, roughly, that being a possible world is being an element of some model which does not contravene the modal facts. ${ }^{10}$

\footnotetext{
${ }^{9}$ Throughout this section, I assume that our uninterpreted formal language is a first-order modal language with quantification, identity, and individual constants. This extends the treatment at (Kripke, 1963, pp. 84, 90), which omits the treatment of individual constants. Such a language may be inadequate to express the full panoply of modal facts; see $\S 5$ below.

${ }^{10}$ If it turns out that Kripke-models are not suitable to represent the modal facts, because, say, identity turns out to be contingent, then we will have to use some other sort of model instead, e.g., the counterpart-theoretic models of (Lewis, 1968) or (Hazen, 1979).
} 


\subsection{Interpreted Models}

What are we to make of the idea of such a model "contravening the modal facts?" It might be thought that the very idea that a model, a mathematical entity of a formal model theory, contravenes the modal facts is confused. Models don't have anything to say about modal facts. The "predicates", "names", and "sentences" which are assigned values in a model are sequences of uninterpreted formal strings, defined in abstraction from any natural language in which we express facts.

It is plausible to argue that we get contact with the modal facts only if we suppose that the model theory somehow gives the semantics of some fragment of, say, English, or at least an analysis of the facts reported by truths expressible in that fragment of English. But accepting that the model gives the semantics of some fragment of English would require that we accept that the real truth-conditions of such English sentences as "it is possible that Bush lose the 2000 electoral vote" should be given in terms of truth in some model. It is surely implausible to suggest that these English sentences have truth-conditions involving such model-theoretic entities as the members of the set $W$ of some model.

This objection relies on the idea that that the only way model theory could bear on the modal facts is by providing a semantics or analysis for the English vocabulary used to state those facts. Our present aim, however, is a theory of possible worlds, not a semantics or analysis of ordinary English vocabulary. Primitivism opens the way to denying that the possibility that Bush have lost the 2000 electoral vote needs interpretation. The modal facts reported by English sentences like "Bush might have lost the 2000 electoral vote" can look after themselves, and stand in no need of analysis in other terms; likewise, there is no pressing need to specify such a sentence's truth-conditions in terms of quantification over possible worlds. Theories invoking possible worlds can be useful and insightful, even when they take the modal vocabulary of natural language as basic. This is the point of view we will be exploring. ${ }^{11}$

The original problem still confronts us. How are we to explain the notion of

\footnotetext{
${ }^{11}$ Other primitivists might accept the notion of being a possible world as an unanalyzed bit of modal vocabulary, and insist that other sorts of modal facts have truth conditions stateable in terms of this more basic notion. The point of the paragraph is that the demand that possible-worlds theory provide an analysis in terms of possible worlds of facts reported in more familiar modal vocabulary can be resisted if you do not already accept that modal claims in general stand in need of analysis in non-modal terms.
} 
a model's "contravening the modal facts?" We need a way to interpret models, so that they say something about the modal facts. To start with, we will need a key, a way of matching the predicates and names of the uninterpreted formal language with properties and entities. The key will not provide the means of interpreting English claims or modal facts in terms of the extensional model theory. Rather, the key provides the means of interpreting the sentences of the hitherto uninterpreted object language of the model back into English (or some other natural language which we could understand). We don't use the model to help us understand what the English claims; we use the English to help us understand what the model "claims."

Call a key acceptable iff it maps '=' to identity, assigns a formal predicate to every property expressible in English, ${ }^{12}$ and assigns an individual constant to every designee of an English name. ${ }^{13}$ An acceptable key provides us with everything we need to interpret the formal language of a Kripke-model. We can "read out" any sentence of a formal language whose predicates and terms are all mapped by the key to properties and entities expressible in English. For instance, if $t$ is mapped to George Bush, and $F$ is mapped to the property designated by "lost the 2000 electoral vote", then " $\nabla F t$ " can be read as "it is possible that Bush lost the 2000 electoral vote."

Where a key maps one or more of the predicates or terms of a formal sentence to properties or entities not designated by any English expression, there will be no reading in English. But the key does give us the means to recursively define an interpretation for the sentence. The general idea is that open sentences are interpreted as complex predicates expressing relations, and then their closures under existential quantification are interpreted as expressing the claim that there are things that stand in the relevant relation. For instance, " $(F x \wedge G y)$ " is interpreted as the relation that any pair of objects stands in just in case the first member of the pair is $F$ and the second member of the pair is $G$. " $(F x \wedge G x) "$ is interpreted as the 1-place relation that any object stands in just

\footnotetext{
${ }^{12}$ This formulation may verge on paradox, if, for instance, there is such a property as being heterological, and this property is expressible in English. On its own, however, the formulation is not paradoxical.

${ }^{13}$ If the restriction to English is deemed too parochial, we may stipulate instead that the key maps formal predicates to those individuals and properties picked out by any natural language whatsoever. A particular language in the class of formal languages I am treating (see n. 9 above) may be specified by its stock of non-logical predicates and constants. Thus, the set of languages interpreted by a given key $K$ can be read off of the stock of predicates and constants in the domain of $K$.
} 
in case it is both $F$ and $G$. " $(\exists x)(F x \wedge G x)$ " is interpreted as the claim that there is something that is both $F$ and $G$. The interpretations for negations, conjunctions, and necessity-claims are given in the obvious way. ${ }^{14}$

Call the pair of a model $M$ and an acceptable key $K$ an interpreted model. We can now define the notion of an interpreted model's contravening the modal facts. An interpreted model $\langle M, K\rangle$ contravenes the modal facts iff there is some $\phi$ true in $M$, but whose interpretation according to the key $K$ is not true. Some of the interpretations of sentences true in a model $M$ will make straightforwardly modal claims. For instance, there is an acceptable key according to which " $\nabla F t$ " can be interpreted as the claim that it is possible that Bush lose the 2000 electoral vote. The truth, or lack thereof, of such a modal claim is itself a modal matter. Hence it will be a modal matter whether $\langle M, K\rangle$ contravenes the modal facts.

I will say that something is an element of the interpreted model $\langle M, K\rangle$ if it is an element of $M$. A precise statement of the thesis, then, is that being a possible world is being an element of an interpreted model $\langle M, K\rangle$ which does not contravene the modal facts. ${ }^{15}$ There is a corresponding notion of what it is for there to be a possible world at which some claim is true: roughly, there is a world at which $S$ is true iff a formal sentence that $S$ interprets is true at some element of some interpreted model which does not contravene the modal facts. ${ }^{16}$ Call this the interpreted models conception of possible worlds.

\subsection{The Three Constraints Satisfied}

This explanation of the notion of possible world appears to meet the three constraints. Possible worlds exist if the model-theoretic entities of the requisite sort do. The existence constraint seems thereby to be met. At the very least, we pass the buck for meeting the existence constraint for possible worlds to meeting a similar existence constraint for the model-theoretic entities. If one thinks that model theory is a useful fiction, for instance, then the present view may not meet the existence constraint. Taking the model theory at face value, however, secures the existence of possible worlds on the interpreted models conception.

The interpreted models conception is also designed to be modally modest.

\footnotetext{
${ }^{14}$ See Appendix A for details.

${ }^{15}$ Here and for most of what follows I will ignore complications required to take account of the accessibility relation $R$.

${ }^{16}$ See Appendix B.
} 
Notice that, unlike some possible worlds theories, what it takes to be a possible world is explicitly explained in terms of the modal facts - exactly the sorts of facts reportable by instances of (SC)'s left-hand side. In essence, the present view reverses the interpretation of (SC) favored by those who propose to use it to analyze modal facts in terms of possible worlds. ${ }^{17}$ On the interpreted models conception, what it takes to be a possible world is explained in terms of what might have been the case. Thus, the modal facts determine which possible worlds there are.

Finally, consider the requirement of ontological modesty. Possible worlds on the interpreted models conception are just whatever sort of entities are appealed to in ordinary, mathematical model theories. Their metaphysical status and nature are no more extravagant than any other entities appealed to in model theory, for instance, of first-order logic. Importantly, we make appeal to no such entities as, e.g., Barack Obama's possible seventeenth granddaughter.

\subsection{Menzel's Theory}

The interpreted models conception is close in spirit to one offered by (Menzel, 1990). Though the views differ on many details, they share a common focus on the interpretation of models. Nevertheless, the view I have described enjoys two advantages over Menzel's proposal, one philosophical, one technical. The philosophical advantage first: Menzel repudiates the constraint of existence, claiming that there are no non-actual possible worlds. ${ }^{18}$ From the present point of view, this rejection of possible worlds is both regrettable and unnecessary. It is regrettable because it requires rejecting most instances of (SC), implying that the uses made of possible worlds by end-users must be rejected if they are to be taken at face value. Rejecting possible worlds is unnecessary because the interpreted models conception shows how they can be accommodated without sacrificing modal or ontological modesty.

Menzel's rejection of possible worlds might be defended by claiming that the uses of apparent quantification over possible worlds in end-users' theorizing is a mere façon de parler. According to the defense, end-users aren't really

\footnotetext{
${ }^{17}$ Here I principally have in mind the theories of (Carnap, 1947) and (Lewis, 1986). Thanks to an anonymous referee.

${ }^{18}$ (Chihara, 1998) also has a similar view, though his view may fail to meet modal modesty, by requiring the truth of all axioms of S5. For an argument that the axioms of S5, even if true, are not logically true, see (Salmon, 1989).
} 
committing themselves to the truth of instances (SC) and the corresponding existence of possible worlds; what they say can be unpacked in such a way that what it expresses are the very model-theoretic facts in virtue of which possible worlds exist and satisfy instances of (SC) according to the interpreted models conception. ${ }^{19}$ Despite appearances, for instance, end-users don't really commit themselves to the claim that it is possible that Bush lost in 2000 iff there is a possible world at which 'Bush lost the 2000 electoral vote' is true; they only commit themselves to the claim that it is possible that Bush lost in 2000 iff there is an interpreted model of the appropriate sort that fails to contravene the modal facts.

We may differ about how serious a cost in plausibility is incurred by this proposed reinterpretation, but there is little doubt that there is some cost. End-users certainly seem to be committing themselves to the truth of (SC). It is better to interpret their theories at face value unless there is compelling reason to do otherwise. But, I have argued, the interpreted models conception shows that neither modal nor ontological modesty provide any such reason. Since there's no need to reinterpret end-users' apparent quantification over possible worlds, it's better to take it at face value. In this respect, then, the interpreted models conception has an advantage over any view which, like Menzel's, denies the existence of possible worlds.

Now for the technical advantage. Associated with each possible world in a Kripke-model for a modal language is a Tarski-style model for a non-modal predicate calculus. Each world has a domain over which quantifiers range, and corresponds to an assignment of extensions to all of the predicates and terms. ${ }^{20}$ Menzel suggests, in effect, that such a Tarski-model represents a possibility in virtue of a certain modal fact: the model has an interpretation on which it could have been an accurate representation of the way things are. ${ }^{21}$ For instance, a Tarski-model which has an interpretation on which Gore is in the extension of "won the 2000 electoral vote" could have been an accurate representation of how things are; if Gore had won, that model, so-interpreted, would have been accurate. But now there is a problem: some modal claims themselves concern

\footnotetext{
${ }^{19}$ See (ISC) in Appendix B below for a concise statement of the relevant correspondence between the modal and model-theoretic facts. Thanks to an anonymous referee for suggesting this avenue of response.

${ }^{20} \mathrm{I}$ am ignoring for the purposes of the present discussion the fact that a Kripke-model may assign to a term a value outside the domain of a given world.

${ }^{21}$ See esp. (Menzel, 1990, p. 371).
} 
further possibilities. Take, for example, the essentialist claim that humanity is a necessary feature of anything that has it; and, further, that this fact is not just contingent, but necessary. Imagine now two Tarksi-models. One of those models (on a given interpretation) represents a possibility in which there are more humans than there actually are, by including an entity in the extension of "human" which does not represent any actual human being, though it could have represented some non-actual human being. The other model (on the same interpretation) represents a possibility in which there are more turnips than there actually are by including the very same entity in the extension of "turnip." Both models could have been accurate representations of how things are nonmodally. But they don't "fit together" on our essentialist assumption, since, taken together, they jointly represent the claim that there could have been something which is contingently human. ${ }^{22}$ So, on pain of modal extravagance, Menzel needs a way to "fit together" the interpreted non-modal models so that these kinds of essentialist claims are accommodated. The problem engenders quite a bit of complication in Menzel's proposal. ${ }^{23}$

This problem is avoided entirely by the interpreted models conception. The starting point for the interpreted models conception are interpreted Kripkemodels whose embedded Tarski-models collectively are an accurate representation of modal reality. Assuming that modal reality can be modeled using the apparatus of possible worlds, the "fit" among the embedded Tarski-models required to accommodate essentialist claims is thereby guaranteed without further complication. If an interpreted model contravenes some essentialist truth, then its elements are not possible worlds on the interpreted models conception. Thus, the interpreted models conception avoids the complications attending Menzel's proposal.

\section{Being a Possible World is a relational condi- tion}

A couple of problems for the interpreted models conception suggest themselves immediately. On the interpreted models conception, being a possible world is a

\footnotetext{
${ }^{22}$ For Menzel's full treatment of this and related problems, see (Menzel, 1990, pp. 373-80).

${ }^{23}$ See, for instance, the condition $\left(C_{n m}\right)$, characterized as "admittedly hairy" by Menzel, on (Menzel, 1990, p. 379).
} 
relational condition. Something is a possible world only if there is an interpreted model to which it bears a certain relation. This gives rise to two problems.

\subsection{Is Everything a Possible World?}

First, since model theories are typically silent on the underlying nature of the entities they quantify over, there seems to be no bar to any individual's being a possible world. If there is any interpreted model at all that fails to contravene the modal facts, then for any given individual there is an interpreted model that fails to contravene the modal facts and has that individual as an element. So Bush is a possible world, as are you and I, the Antarctic ice sheet, the square root of 2 , etc.. Some commentators have suggested that this presents a problem for, or even a refutation of, the interpreted models conception. ${ }^{24}$

The fact that the interpreted models conception implies that everything is a possible world would indeed pose a problem, if it were intuitively evident that some things are not possible worlds. Some theories, ${ }^{25}$ together with evident facts, recommend this opinion. And perhaps some theorists would be surprised to discover that absolutely everything is a possible world. But no one should think it intuitively evident that some individuals are not possible worlds. Once we have convinced ourselves that the notion of a "possible world" is a technical notion, we should be no more surprised to discover that everything is a possible world than we are surprised to discover, e.g., that everything is an element of some boolean algebra.

Still, it is important to distinguish the technical claim that everything is a possible world from an apparently non-technical claim expressed by similar verbiage. There are, we have seen, ordinary English constructions of a form apparently similar to "possible world" that make perfectly good sense, e.g., "possible 2012 Republican presidential nominee". Suppose we take "possible world" as a bit of ordinary English on this model. So-interpreted, the claim, "everything is a possible world" is false, since, for instance, Bush is not such that it is possible that he be a world. The interpreted models conception does not say otherwise. I have only claimed that this is not the correct way to take possible worlds claims as they occur in theorizing about, e.g., the metaphysics of mind or

\footnotetext{
${ }^{24}$ The need to address this problem was pressed independently by Michael Fara and Harold Hodes.

${ }^{25}$ See (Lewis, 1986), (Plantinga, 1973), and (Stalnaker, 1976).
} 
morality, epistemology, linguistics, etc. I think it would pose a serious problem for a theory if it implied this ordinary English claim; but the interpreted models conception does not.

Some theorists will still object to the deflationary upshot of the interpreted models conception. According to the interpreted models conception, a possible world is just any old thing (or any old pure set) that plays a certain role in a certain kind of model. Surely, the objection goes, we have an intuitive, if somewhat inchoate, conception of what possible worlds are: they are complete ways things could have been. This intuitive conception motivates the endorsement of (SC); it is what makes the further theorizing by end-users seem promising. But the interpreted models conception does not underwrite this intuitive conception. So, the objector concludes, the interpreted models conception renders (SC) unattractive and fails to capture what seemed so promising about theorizing that uses possible worlds.

I myself have no very strong attachment to the conception of possible worlds as complete ways things could have been. Does rejecting that admittedly popular conception in favor of the interpreted models conception rob possible worlds of their utility for theorizing by end-users in other fields? To my mind, the answer is clearly 'no': the interpreted models conception does not undermine the utility of possible worlds for theorizing by end-users. In my view, what makes (SC) attractive is that it has been theoretically useful, if not for giving a reduction of modal facts or modal discourse, then for a host of other enterprises. The usefulness of possible worlds for such theorizing does not depend on giving an account of their nature that verifies the particular conception to which the objector appeals.

Still, it might be contended that if there are any worlds at all, there must be some feature that demarcates them from non-worlds, even if there's no reason to cleave to any particular conception of their nature. That is, if there are any possible worlds, then there must be some feature that's distinctive of them, and so possessed by all the worlds and lacked by at least some non-worlds. Metaphysicians differ on the nature of possible worlds, but they have until now agreed that there is some feature shared by possible worlds and lacked by nonworlds. As I have noted, however, there is no such feature on the interpreted models conception. Thus, the objection goes, the interpreted models conception is a view on which there aren't really any possible worlds; there is only a 
certain model-theoretic role - a role which anything can play. For this reason, the objector concludes, the interpreted models conception does not satisfy the existence constraint after all. ${ }^{26}$

The objection relies on the contention that the existence of possible worlds requires there to be a feature which possible worlds have and which some nonworlds lack. There is no reason to accept this contention. On the interpreted models conception being a possible world is a relational condition. It does not follow that there are no worlds. Being not made of phlogiston is also a relational condition, and one that is satisfied by everything. We should not conclude on this basis that there aren't really any things not made of phlogiston, nor that there is only a certain scientific role - a role which anything can (and does) play. Similarly, the fact that being a possible world is a relational condition does not imply that there aren't really any worlds. It does imply that there is no "intrinsic nature" that makes something a possible world. It also implies that possible worlds are not an identifiable sub-category of entity. Thus, there is less to say about the distinctive nature of possible worlds in the interpreted models conception than there is on other theories of possible worlds. But any theory of possible worlds will have as an upshot that all competing theories of possible worlds are wrong in some respect, so there's no special grounds for rejecting the interpreted models conception on this score.

\subsection{Relations Among Worlds}

The fact that being a possible world is a relational condition also introduces a second problem, encountered when attempting to discern the features of possible worlds. We may want to know whether a certain relation obtains among worlds. For instance, we might want to know whether there are at least two possible worlds. There will be two ways to take this question on the interpreted models conception. On one way of taking this question, a 'yes' answer requires that there be a single interpreted model with at least two elements. On another way of taking this question, a 'yes' answer can also be given when there are two interpreted models each with only a single element. This means that sometimes questions about the features of possible worlds lack a single, determinate answer, contrary to what we might have hoped or expected.

\footnotetext{
${ }^{26}$ Thanks to an anonymous referee for suggesting the need to respond to these last two concerns.
} 
It seems to me that, though we must acknowledge that this is a problem, we should not make too big a deal out of it. We encounter a similar problem with respect to other relational conditions. For instance, being a parent, like being a world, is a relational condition. Someone is a parent only if there is an individual to whom she bears a certain relation. There will sometimes be a problem when attempting to discern features of parents.

Imagine, for instance, a village in which every pair of parents has only one child, and that each member of each pair always agrees with the other. We can take the question, "Do parents ever disagree?" in two ways. On one way of taking it, the coordinated interpretation, the answer is 'no', since parents of a given child always agree. On the other way of taking the question, the uncoordinated interpretation, the answer may still be 'yes', since parents of different children may disagree. Also, sometimes on the coordinated interpretation of a question about the features of parents, the right first response to a yes-no question is, "it depends." Suppose, for instance, that a new family moves into the village, and that the parents of this family disagree on some matters. Consider the question, "Do parents disagree?" On the coordinated interpretation of this question, the right first response is, "it depends on which parents are under discussion."

The problem of disentangling the coordinated and uncoordinated interpretations of these questions is handled without significant misunderstanding in most cases. Where there is misunderstanding, it can usually be corrected by delineating which interpretation is salient. A similar strategy should work for problems understanding claims regarding the features of possible worlds. In the discussion that follows, I always intend the coordinated interpretation of questions about worlds; that is, I intend to address questions about whether there is a single interpreted model in which worlds have the requisite features.

If there are any interpreted models which do not contravene the modal facts, then there will be non-isomorphic models which do not contravene the modal facts. Some of these models will deliver different answers to the question, "How many possible worlds are there?"27 The appropriate response to this difficulty is to acknowledge it, but not to overestimate its importance. Suppose that we are given two interpreted models, neither of which contravenes the modal facts,

\footnotetext{
${ }^{27}$ For a quick and dirty illustration, assume that $\langle w, W, R\rangle$ is the model structure for some interpreted model $M$ which does not contravene the modal facts. Just add to $W$ all the members of some strictly larger set, making sure that in the new model, each new element is a "copy" of some old element, along the lines suggested in the next paragraph.
} 
but which contain different numbers of possible worlds. Whatever else there may be to say about such models, they agree in all respects on those aspects of modal reality which they model. A difference on the question of how many worlds there are appears to make no difference to the correct description of what might have been the case.

The view at hand makes short work of the question of whether there are distinct worlds that are indiscernible with respect to which claims are true in them. Suppose we are given an interpreted model which does not contravene the modal facts. Now just create a new model by adding an element $w^{*}$ to the set of worlds of the old model, so that $w^{*}$ is a "copy" of some world $w$ in the old model. The extensions of terms and simple predicates in $w^{*}$ are exactly the same as in $w$, and exactly the same worlds are accessible from, and to, $w^{*}$. The new interpreted model will verify exactly the same claims as the old, and so will not contravene the modal facts. So there is a model in which there are distinct worlds that are indiscernible in the relevant sense. Some may see this as a problem with the account. I think of it instead as an interesting (and

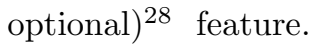

In summary, these questions involving the features of possible worlds pose no serious problems.

\section{$5 \quad$ Expressibility Problems}

One problem for the interpreted models conception involves limitations on which modal facts can be expressed using primitive modal vocabulary. A language containing quantifiers over worlds is expressively more powerful than a language containing only sentential modal operators. In particular, it is possible to express certain relations between worlds in a language with quantifiers over worlds that cannot be expressed in QML. For instance, one cannot express in QML the claim that there is a world $w^{\prime}$ such that every world $w$ contains some individual also contained in $w^{\prime} .{ }^{29}$ Let's suppose, for simplicity, that all modal claims stateable in a primitive modal vocabulary are representable in QML. Then the primitive modal vocabulary has less expressive power than a language containing quantifiers over worlds. I have been suggesting, in effect, that this extra

\footnotetext{
${ }^{28}$ We can rule out distinct, indiscernible possible worlds by requiring that every pair of worlds differ with respect to domain or assignment of extensions to non-logical vocabulary.

${ }^{29}$ (Hazen, 1976)
} 
expressive power is wasted. Insofar as such questions are not settled by those modal facts expressible in a primitively modal language, we need not worry too much about which answers come out.

We have supposed that all modal claims stateable in a primitive modal vocabulary are representable in QML. But some theorists have argued, independently of any theory of possible worlds, that there is more to modal reality than can be said using the sentential modal operators, the "boxes and diamonds", characteristic of QML. ${ }^{30}$ Let's suppose now that these theorists are correct. There will then be another failure of expressive power that cannot be so quickly dismissed. Consider for illustration the argument of (Lewis, 1986, pp. 13-17) that some supervenience claims cannot be expressed in the idioms representable in QML. Let's focus in particular on the claim that psychological facts supervene on physical facts. Lewis gives a vague but intuitive gloss of this claim in explicitly modal terms: there could be no psychological difference without a physical difference. ${ }^{31}$ He argues that this "no difference" claim is not implied, and hence not adequately represented, by the claim we get using only a sentential modal operator: "it is not possible that two things should differ with respect to their psychological properties but not with respect to their physical properties". (The problem is that the "no difference" claim Lewis intends fails if there are two individuals of different worlds which are psychologically different but physically the same, while the proposed "box and diamond" gloss need not fail.) Rather than focus on whether someone might offer an alternative "box and diamond" gloss of the "no difference" claim that more adequately captures its import, let's just suppose that Lewis is right: there are two interpreted models that do not contravene the modal facts in the sense under discussion, and so agree on all the "box and diamond" modal facts, but that differ with respect to other modal facts. In particular, let's suppose that there are two such interpreted models which differ with respect to supervenience facts.

This supposition gives rise to a serious objection. I have been claiming that many questions regarding worlds, including how many there are, whether there are distinct but indiscernible worlds, and so on, are to be answered, "it depends on which interpreted model the worlds are in." I have suggested that there is no

\footnotetext{
${ }^{30}$ (Lewis, 1986, pp. 13-17).

${ }^{31}$ Let's ignore the problem of saying what counts as a physical difference. Let's also ignore the interesting differences between alternative modal characterizations of supervenience relations; see (Teller, 1984). The comments in the main text should apply equally well to all of the alternatives.
} 
pre-theoretical reason to believe that this is the wrong way to respond. But now consider the question of whether psychological facts supervene on physical facts. Surely, the objector urges, the question of whether the supervenience relation holds is a matter of modal fact. We may not know the right answer, and there may be difficulties interpreting the claim, but at least we know that "it depends on the model" is wrong. According to this objection, our proposed explanation of the notion of a possible world does not meet the constraint of modal modesty: it requires that we give an evidently incorrect response to questions regarding supervenience relations.

The objection relies the assumption that there are two models which do not "contravene the modal facts" in the sense we have given to that expression, but which deliver different answers to supervenience questions. This assumption requires that each of our two interpreted models delivers an answer to supervenience questions, and that for some such questions, they deliver different answers. An interpreted model can only deliver an answer to a supervenience question if there is some way of interpreting that question in terms of some feature of the interpreted model. So the objection assumes what we might call the interpretability of supervenience: that there is a way of interpreting supervenience questions in terms of some feature of the interpreted model.

For instance, an interpreted model might be thought to deliver a "no" to the question of whether psychological facts supervene on physical facts in virtue of containing two worlds which assigned the same extensions to predicates mapped by the key to physical properties, but assigned different extensions to predicates mapped by the key to psychological properties. Another interpreted model might be though to deliver a "yes" answer to the same question in virtue of containing no such pair of worlds. ${ }^{32}$ But if models are interpretable in this way, then we have evidently drawn the notion of "contravening the modal facts" too narrowly: we need only augment the explanation of that notion with a specification of how a model can contravene supervenience facts in addition to contravening "box and diamond" facts. If supervenience facts can be interpreted in any possible worlds theory in terms of which individuals have which properties in which worlds, then the interpreted models conception can use that interpretation to restrict the class of models which do not contravene the modal

\footnotetext{
${ }^{32}$ This would be a plausible interpretation only for a particularly weak brand of supervenience, which has never been explicitly advanced to the best of my knowledge. This toy interpretation is a mere illustration.
} 
facts. And then the original explanation of the notion of a possible world as an element of an interpreted model which does not contravene the modal facts will not have the untoward consequence that there are two such models which differ on matters of supervenience.

Suppose we reject the interpretability of supervenience: interpreted models just have nothing to say about whether there could be a psychological difference without a physical difference. It seems, then, that our theory of possible worlds is ill-suited to modeling supervenience claims. But notice that any interpreted model provides the means of saying which individuals have which properties in which worlds. For instance, an interpreted model, together with a specification of which properties are physical and which are psychological, provides the means of determining whether there is anything which has exactly the same physical properties, but different psychological properties in two worlds of the interpreted model. In this respect, our interpreted model can say as much about what possible worlds there are and what their denizens are like as any other possible worlds theory. So, rejecting the interpretability of supervenience implies that supervenience claims are not interpretable in terms of any theory of possible worlds. If this is a strike against the interpreted models conception, it is equally a strike against any other theory of possible worlds.

The point generalizes. Any aspect of modal reality is either interpretable in terms of features of worlds and what goes on in them or it is not. If it is

interpretable in such terms, then we can use the interpretation to express a constraint that a model must meet in order not to contravene the modal facts. If it is not interpretable in such terms, then this is a weakness of possible-worlds approaches generally, and so provides no reason to favor some alternative theory of possible worlds over the one we have been discussing.

\section{The Point of Possible Worlds}

The interpreted models conception is frankly reductive: the notion of being a possible world is explained in terms of what the possibilities are. This stands the standard possible worlds reduction of necessity and possibility on its head: the facts regarding worlds are explained in terms of the possibilities, rather than vice versa.

Some will complain, then, that the point of adopting a theory of possible 
worlds has been lost: we adopt a theory of possible worlds to provide an analysis of either the modal facts or our modal concepts in terms of ordinary quantification over extraordinary entities. Further, possible worlds, on the interpreted models conception, will not play any role in the explanation of modal principles. For instance, we can't use quantifier negation laws to explain why the necessity of a claim is equivalent to the impossibility of its negation. On the interpreted models conception, the order of explanation is reversed. For instance, the fact that such modal principles hold explains the fact that "Socrates is human" is true at every possible world just in case "Socrates is not human" is true at no possible world. On the interpreted models conception, possible worlds play no interesting role in any account of modality or of modal concepts.

If the only point to adopting a theory of possible worlds were to provide such an account, then the objection would be warranted. But it would apply equally well to any other primitivist account of possible worlds. ${ }^{33}$ Since the aim here is not to defend primitivism, but to suggest a theory of possible worlds that should be attractive to primitivists, the objection should be addressed in detail on another occasion.

More significantly, nobody, primitivist or not, should accept that the only point to the invocation of possible worlds is to provide a reduction of modal facts to non-modal facts. As I have emphasized several times already, (SC) and possible worlds have become embedded in rather a wide swath of theorizing by end-users about such (apparently) non-modal matters as the nature of mind, language, morality, and rationality. Possible worlds are tools in a wide variety of other philosophical (and not-so-philosophical) projects. ${ }^{34}$ A theory of possible worlds should illustrate and underwrite their fitness as tools for inquiry in fields far removed from the metaphysics of modality, the analysis of modal concepts, or the semantics of modal discourse. The fitness of possible worlds for theorizing

\footnotetext{
${ }^{33}$ Perhaps a weaker version of the complaint applies to the present view. It is consistent with primitivism that a possible worlds theory that verifies instances of (SC) provides the makings of an analysis of the facts which we can report using the sentential operators "it is necessary that..." and "it is possible that..." in terms of more basic, and still modal, facts regarding possible worlds. The point of possible worlds theories, on this more moderate view, is to analyze these "box and diamond" modal facts. Endorsing the interpreted models conception requires abandoning even this more modest analytical aim. I believe the appropriate response to this complaint is to claim that this aspect of the theory is a feature, rather than a bug. There is no reason to think that the "box and diamond" modal facts are any less fundamental or more problematic than the facts about possible worlds; they seem to stand less in need of analysis than the facts which the more modest view proposes as their ground.

${ }^{34}$ Thanks to Jessica Wilson for discussion.
} 
in philosophy of mind, linguistics, ethics, epistemology, etc., does not hang on their providing the means to analyze modality or explain modal principles.

Our three constraints were motivated by this conception of possible worlds as theoretical tools. Neutrality on modal and ontological matters is desirable because it frees end-users from untoward commitments in ontology or the metaphysics of modality. End-users' theories don't appear to be hostage to the abstruse ontological or modal claims debated among metaphysicians of modality, and a theory of possible worlds shouldn't make them seem so. Similarly, the constraint of existence turns out just to be a matter of interpreting literally and at face value theories that incorporate (SC). The fact that the interpreted models conception meets all three constraints is therefore a conspicuous virtue of the account. Possible worlds are here to stay. I have argued that the interpreted models conception shows how they can be comfortably accommodated. ${ }^{35}$

\section{Appendix A: How to Interpret a Model}

The interpreted models conception presumes that formal Kripke-style models can be interpreted so that they say something about the modal facts, and that, so-interpreted, they can be assessed for accuracy, i.e. for whether they "contravene the modal facts." In Kripke-style model theories the formal sentences of QML are interpreted using a standard apparatus of assignments of values to variables. The familiarity of this standard apparatus gives rise to a natural worry for the interpreted models conception. If we use the standard apparatus of Kripke-style semantics in our attempt to interpret what a formal model says about the real modal facts, then, one might worry, we will have to find "real" counterparts for the model-theoretic indices: real possible worlds in which sentences will be evaluated, and real possibilia to assign as values to the variables. It is difficult to see how such an interpretation can avoid ontological extravagance.

Fortunately, we can set this worry aside. There is another way to interpret the formal sentences of QML, which does not use the standard Kripke-style apparatus of assignments of values to variables. Thus, we can interpret QML

\footnotetext{
${ }^{35}$ Thanks to Roberta Ballarin, Ben Caplan, David Christensen, Tyler Doggett, Michael Fara, Harold Hodes, Paul Hovda, Mark Moyer, Derk Pereboom, Guy Rohrbaugh, Jessica Wilson, an anonymous referee, and an audience at Dartmouth College for invaluable comments and criticism.
} 
without any appeal to features of possibilia. Instead of giving an interpretation of the open formal sentences of QML in terms of satisfaction under assignments of values to variables, we can interpret those sentences as complex predicates, expressing properties and relations. ${ }^{36}$ Correlatively, the free variables of a formula are thought of as syntactic markers of argument-places for the relation expressed by the formula, rather than as terms which refer (on an assignment). The technical trick which allows us to interpret the formal sentences of QML without ontological extravagance is the trick of recursively assigning relations to wffs of QML as their interpretation. (We think of properties as 1-place relations, and claims as 0-place relations.)

The recursion, however, is not directly on the complexity of the formula. We will need to assign different relations to $F x \Leftrightarrow F x$ and $F x \Leftrightarrow F y$, even though we assign the same relations to $F x$ and $F y \cdot{ }^{37}$ Without fancy footwork, recursion on the complexity of a formula will not allow this. Instead, we will use some novel syntactic notions to carry out the idea.

The use of individual constants introduces some complications. I will therefore start by showing how to give interpretations for constant-free formulae. The idea of our novel syntax is to think of argument-places for relations as successively "coordinated" by replacement of free variables. For instance, $F x \Leftrightarrow F x$ is obtained from $F x \Leftrightarrow F y$ by "coordinating" the two argument places marked by $x$ and $y$. The result of a coordination of a formula is its "coordinand", and uncoordinated formulae are called "clumsy." More canonically:

A formula $\phi$ is the $x_{n}-x_{m}$ coordinand of $\psi$ iff:

i. $x_{n}$ occurs free in $\psi$;

ii. $x_{m}$ occurs free and exactly once in $\psi$;

iii. $x_{n}$ does not occur after $x_{m}$ in $\psi$;

iv. $x_{n}$ is free for $x_{m}$ in $\psi$; and

v. $\phi$ is the result of replacing the only occurrence of $x_{m}$ in $\psi$ with $x_{n}$.

A formula $\phi$ is clumsy atomic iff it is $F^{n} x_{1}, \ldots, x_{n}$, for $n$ distinct variables $x_{1}, \ldots, x_{n}$.

A formula $\phi$ is a clumsy conjunction if it is a conjunction whose conjuncts share no free variables in common.

\footnotetext{
${ }^{36}$ The treatment given here bears a significant resemblance to the methods of (Quine, 1960) and (Bealer, 1983).

${ }^{37}$ Fine's worry in (Fine, 2003a) about the tenability of an ordinary satisfaction-based semantics is related to this point. I originally came to the sort of semantics on offer here as a result of reflection on Fine's puzzle.
} 
We can think of a formula as being built up by coordination, negation, etc. from clumsy constituents. We obtain formal specification of this construction by defining the notion of a coordination analysis for a formula.

A coordination analysis for a formula $\phi$ is a tree such that:

i. $\phi$ is the root node;

ii. all leaves are clumsy atomic formulae;

iii. every node has at most two children;

iv. if a node $\phi$ has two children $\psi_{1}$ and $\psi_{2}$, then $\phi$ is the clumsy conjunction $\left(\psi_{1} \wedge \psi_{2}\right)$;

v. if a node $\phi$ has exactly one child $\psi$, then either:

a. $\phi$ is $\neg \psi$;

b. $\phi$ is $(\exists x) \psi$;

c. $\phi$ is $\square \psi$; or

d. $\phi$ is the $x_{n}-x_{m}$ coordinand of $\psi$;

It can be shown that every formula $\phi$ has a coordination analysis by a somewhat tedious induction on the complexity of $\phi$. The two interesting cases are when $\phi$ is atomic, and when $\phi$ is a conjunction. For the atomic case, since leaves must be clumsy atomic formulae, we must show how an arbitrary coordinated atomic formula can be analyzed in terms of step-wise coordination of argument-places. Similarly, for the case of conjunction, we must show how any conjunction can be analyzed in terms of step-wise coordination from some clumsy conjunction. In both cases, we can prove that $\phi$ has a coordination analysis by induction on the number of distinct free variables in $\phi$, and then by induction on the number of occurrences of the last multiply-occurring free variable in $\phi$.

A formula will generally have many coordination analyses. More than one is more than is convenient, so we should define some nice well-ordering $\mathcal{R}$ on coordination analyses, and then let the coordination analysis of $\phi$ be the $\mathcal{R}$-least coordination analysis of $\phi$.

Recall that an acceptable key $K$ assigns an $n$-ary relation to every $n$-place predicate $F^{n}$, and the identity relation to '='. We will assume that the relations are closed under operations corresponding to the syntactic operations of clumsy conjunction, negation, necessitation, coordination of argument-places, etc. Now we can finally define the interpretation of $\phi$ by $K\left([\phi]^{K}\right)$ by recursion on the depth of the coordination analysis of $\phi$ : 
i. if $\phi$ is clumsy atomic (and so a leaf), then $[\phi]^{K}$ is $K\left(F^{n}\right)$;

ii. if $\phi$ is the $x_{n}-x_{m}$ coordinand of $\psi\left(x_{1}, \ldots, x_{n} \ldots x_{m}, \ldots, x_{j}\right)$, then $[\phi]^{K}$ is the relation $a_{1}, \ldots, a_{n}, \ldots, a_{m-1}, a_{n}, \ldots, a_{j}$ stand in $[\psi]^{K}$;

iii. if $\phi$ is a clumsy conjunction of its children $\psi_{1}$ and $\psi_{2}$ then $[\phi]^{K}$ is the relation
$a_{1}, \ldots, a_{n}$ stand in $\left[\psi_{1}\right]^{K}$ and $b_{1}, \ldots, b_{m}$ stand in $\left[\psi_{2}\right]^{K} ;$

iv. if $\phi$ is the negation of its only child $\psi$, then $[\phi]^{K}$ is the relation $a_{1}, \ldots, a_{n}$ do not stand in $[\psi]^{K}$;

v. if $\phi$ is an existential generalization over $x_{i}$ of its only child $\psi$, then $[\phi]^{K}$ is the relation there is an $a_{i}$ such that $a_{1}, \ldots, a_{i}, \ldots, a_{n}$ stand in $[\psi]^{K}$;

vi. if $\phi$ is the necessitation of its only child $\psi$, then

$[\phi]^{K}$ is the relation it is necessary that $a_{1}, \ldots, a_{n}$ stand in $[\psi]^{K}$.

Adding individual constants is relatively straightforward. Recall that a key $K$ assigns an individual to every individual constant $c$. The individual constants must be ordered, e.g., alphabetically, and we need both to define a way of introducing a constant into a formula, and to extend some of our definitions.

A formula $\phi$ is the $c-x_{m}$ coordinand of $\psi$ iff:

i. $c$ is an individual constant that does not occur in $\psi$; and

ii. $\phi$ is the result of replacing all free occurrences of $x_{m}$ in $\psi$ with $c$.

Add to clause (v.) of the definition of a coordination analysis:

e. $\phi$ is the $c-x_{m}$ coordinand of $\psi$;

Add to the definition of $[\phi]^{K}$ :

vii. if $\phi$ is the $c-x_{m}$ coordinand of its only child $\psi\left(x_{1}, \ldots, x_{m}, \ldots, x_{n}\right)$, then $[\phi]^{K}$ is the relation $a_{1}, \ldots, a_{m-1}, K(c), \ldots, a_{n}$ stand in $[\psi]^{K}$

Notice that constants, unlike variables, are added "all at once;" this measure simplifies the proof that every formula has a coordination analysis (in the new sense). The old proof establishes that every constant-free formula has a coordination analysis. We complete the new proof by supplementing the old proof with inductions on the number of constants in $\phi$ in both the atomic and conjunction cases. 


\section{Appendix B: How to Interpret (SC)}

I have been focused for the entirety of this paper on giving a theory of possible worlds that meets the constraints of existence, ontological modesty, and modal modesty. I have not been directly concerned with providing an analysis or interpretation of (SC), beyond specifying what it takes for something to be a possible world. There is, however, a natural interpretation available, which can be reached in one of two ways. Both ways use the notion of an (English) sentence $\phi$ 's interpreting some formal sentence $\psi$ according to a key $K$. I take the notion of an (English) sentence's expressing a claim as primitive. ${ }^{38}$ Then, $\phi$ interprets $\psi$ according to $K$ iff $\phi$ expresses the claim assigned to $\psi$ by $K$. Call the formal sentence interpreted by $\phi$ according to $K \phi_{K} \cdot{ }^{39} \quad$ Also, for the sake of brevity, I will call an interpreted model good iff it fails to contravene the modal facts.

The first way to our natural interpretation of (SC) is to define all at once the relation $w$ is a possible world at which $\phi$ is true:

$(\mathbf{P W}+\mathbf{T A}) w$ is a possible world at which ' $\phi$ ' is true iff there is a good interpreted model $\langle M, K\rangle$, such that $(i) w$ is an element of $\langle M, K\rangle$, and (ii) $\phi_{K}$ is true at $w$ in $M .^{40}$

Substituting the right-hand side of $(\mathrm{PW}+\mathrm{TA})$ in for its left-hand side in a colloquial variant of (SC):

(CSC) it is possible that $\phi$ iff there is a $w$ such that $w$ is a possible world at which ' $\phi$ ' is true.

yields the interpretation of (SC):

(ISC) it is possible that $\phi$ iff there is a $w$ such that there is a good interpreted model $\langle M, K\rangle$, such that $(i) w$ is an element of $\langle M, K\rangle$, and $(i i) M \vDash_{w} \phi_{K}$.

Given (PW+TA), the interpreted models conception of being a possible world is a natural upshot:

\footnotetext{
${ }^{38}$ If we think of the substituends for $\phi$ in (SC) as claims, rather than natural language sentences, then we should say that every claim expresses itself, and a claim $\phi$ interprets $\psi$ according to $K$ iff $K$ assigns $\phi$ to $\psi$.

${ }^{39}$ Technically, $\phi$ can interpret many sentences according to $K$. If so, let $\phi_{K}$ be the least sentence (according to some well-ordering) that $\phi$ interprets. This wrinkle will not matter for our discussion.

${ }^{40}$ In what follows, let ' $M \vDash_{w} \phi_{K}$ ' abbreviate (ii).
} 
(PW) $w$ is a possible world iff there is a good interpreted model $\langle M, K\rangle$ such that $w$ is an element of $\langle M, K\rangle$.

This way of arriving at an interpretation of (SC) presents (PW) as an upshot, rather than a necessary input, of the interpretation. This is not the most natural way to present it. It is more natural to offer a definition of the relation $\phi$ is true at $w$ that can be used along with (PW) to yield an interpretation of (SC). The second, less direct way of reaching (ISC) follows that more natural course. Here's a definition of $\phi$ is true at $w$ consilient with the interpreted models conception:

(TA) $\phi$ is true at $w$ iff there is a good interpreted model $\langle M, K\rangle$ such that $M \vDash_{w} \phi_{K}$.

Plugging the right-hand sides of (PW) and (TA) into the requisite positions in (SC) yields:

(ISC)* it is possible that $\phi$ iff there is a $w$ such that $(i)$ there is a good interpreted model $\langle M, K\rangle$ that has $w$ as an element, and (ii) there is a good interpreted model $\left\langle M^{\prime}, K^{\prime}\right\rangle$ such that $M^{\prime} \vDash_{w} \phi_{K^{\prime}}$.

(ISC) and (ISC)* are equivalent, since, for any $\phi, M^{\prime} \vDash_{w} \phi_{K^{\prime}}$ only if $w$ is an element of $\left\langle M^{\prime}, K^{\prime}\right\rangle$. So our two ways of interpreting (SC) reach the same destination.

Notice that, according to (TA), for $\phi$ some contingent truth, if $\phi$ is true at $w$, then $\neg \phi$ is also true at $w$. Further, if we adopt a natural definition of the correlative notion of $\phi$ 's being false at $w$ :

(FA) $\phi$ is false at $w$ iff there is a good interpreted model $\langle M, K\rangle$ such that $M \vDash_{w} \neg \phi_{K}$

then it turns out that $\phi$ is both true and false at $w$. Is this objectionable? Not if we remember that being true at $w$, like being a possible world, is a condition that implicitly involves a relation to an interpreted model. As we saw in section 4 above, when we speak of such conditions, what we say is sometimes ambiguous between coordinated and uncoordinated interpretations. As with possible worlds, most of the interesting issues arise with respect to the coordinated interpretation. It's only on an uncoordinated interpretation that a question of the form, "Is $\phi$ both true and false at $w$ ?" should get an affirmative answer. On the more interesting, coordinated interpretation, the answer is clearly no. ${ }^{41}$

\footnotetext{
${ }^{41}$ Thanks to an anonymous referee for suggesting the need for this appendix, and offering some proposals for how the interpretation of (SC) might be carried out.
} 


\section{References}

Frank Arntzenius. Some Problems for Conditionalization and Reflection. Journal of Philosophy, C(7):356-70, July 2003.

George Bealer. Completeness in the Theory of Properties, Relations, and Propositions. The Journal of Symbolic Logic, 48(2):415-26, June 1983.

Karen Bennett. Two Axes of Actualism. The Philosophical Review, 114:297-326, 2005.

Simon Blackburn. Supervenience Revisited. In Ian Hacking, editor, Exercises in Analysis, pages 47-67. Cambridge University Press, Cambridge, 1985.

Rudolf Carnap. Meaning and Necessity: A Study in Semantics and Modal Logic. The University of Chicago Press, Chicago and London, second edition, 1947.

Charles Chihara. The Worlds of Possibility. Clarendon Press, Oxford, 1998.

Kit Fine. Plantinga on the Reduction of Possibilist Discourse. In J.E. Tomberlin and P. van Inwagen, editors, Alvin Plantinga, pages 145-86. Reidel, 1985.

Kit Fine. The Role of Variables. Journal of Philosophy, 100:605-631, 2003a.

Kit Fine. The Problem of Possibilia. In Michael Loux and Dean Zimmerman, editors, The Oxford Handbook of Metaphysics, pages 161-79. Oxford University Press, Oxford, 2003b.

Allen Hazen. Expressive Completeness in Modal Language. The Journal of Philosophical Logic, 5:25-46, February 1976.

Allen Hazen. Counterpart-Theoretic Semantics for Modal Logic. The Journal of Philosophy, 76(6):319-38, June 1979.

Saul Kripke. Naming and Necessity. Harvard University Press, Cambridge, 1980.

Saul A. Kripke. Semantical Considerations on Modal Logic. Acta Philosophica Fennica, pages 83-94, 1963.

David Lewis. Counterpart Theory and Quantified Modal Logic. Journal of Philosophy, 65:113-126, 1968. 
David Lewis. Attitudes De Dicto and De Se. Philosophical Review, 88(4):513-43, October 1979.

David Lewis. On the Plurality of Worlds. Blackwell, Oxford, 1986.

Chris Meacham. Sleeping Beauty and the Dynamics of De Se Beliefs. Philosophical Studies, 138(2):245-69, March 2008.

Chris Meacham. Unravelling the Tangled Web: Continuity, Internalism, NonUniqueness and Self-Locating Beliefs. In Tamar Szabó Gendler and John Hawthorne, editors, Oxford Studies in Epistemology, volume 3. Oxford University Press, Oxford, 2010.

Christopher Menzel. Actualism, Ontological Commitment, and Possible Worlds Semantics. Synthese, 85:355-89, 1990.

Alvin Plantinga. Transworld Identity or Worldbound Individuals? In Milton Munitz, editor, Logic and Ontology, pages 193-212. New York University Press, 1973.

Alvin Plantinga. Actualism and Possible Worlds. In Matthew Davidson, editor, Essays in the Metaphysics of Modality, pages 103-21. Oxford University Press, 2003.

Willard V. Quine. Variables Explained Away. Proceedings of the American Philosophical Society, 104(3):343-7, June 1960.

Gideon Rosen. Modal Fictionalism. Mind, 99:327-54, July 1990.

Nathan Salmon. The Logic of What Might Have Been. The Philosophical Review, 98(1):3-34, 1989.

Theodore Sider. The Ersatz Pluriverse. The Journal of Philosophy, 99(6):279315 , June 2002.

Robert Stalnaker. Possible Worlds. Noûs, 10(1):65-75, March 1976.

Robert Stalnaker. Assertion. In P. Cole, editor, Syntax and Semantics: Pragmatics, volume 9, pages 315-22. Academic Press, Inc., 1978.

Paul Teller. A Poor Man's Guide to Supervenience and Determination. Southern Journal of Philosophy, 22:137-62, 1984. 